\title{
Plasmacytoma of head and neck as initial presentation of multiple myeloma
}

\author{
Rodrigo Jorge de Oliveira', Fabiano Reis' , Atul Kumar Taneja', \\ Luciano de Souza Queiroz², Verônica de Araújo Zanardi ${ }^{1}$
}

45-year-old female with headache and facial tumor growing since 16 months ago. Diagnostic imaging demonstrated a large lesion on the right greater sphenoid wing, extending into the masticator space, nasopharynx, nasal cavity, clivus, sphenoid and maxillary sinuses, and to the anterior and middle cranial fossae.

Histological analysis confirmed the diagnosis of plasmacytoma and later the bone marrow biopsy diagnosed multiple myeloma.

Multiple myeloma compromising the skull base is difficult to diagnose, specially when its onset is a head and neck plasmacytoma, wich is uncommon and overlaps other head and neck tumoral conditions that also present as large extra-axial masses.
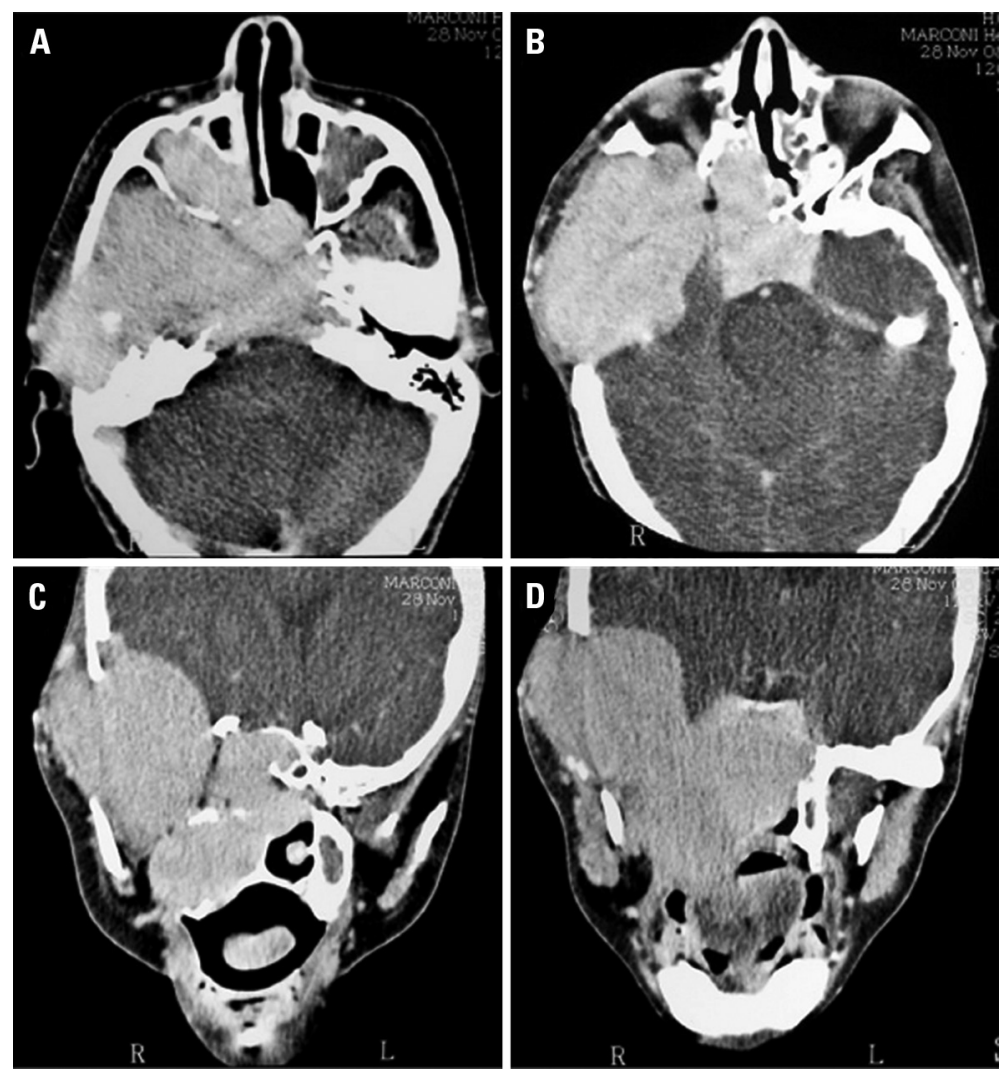

Fabiano Reis

Faculdade de Ciências Médicas Universidade Estadual de Campinas Departamento de Radiologia Rua Tessália Vieira de Camargo 126 13083-887 Caixa Postal 6111 Campinas SP - Brasil

E-mail: fabianoreis2@gmail.com

Fig 1. CT: [A and B] Contrast-enhanced axial, [C and D] Contrast-enhanced coronal. On CT, the lesion presented attenuation similar to soft tissues, with intense and heterogeneous enhancing after contrast .

\section{PLASMOCITOMA DA CABEÇA E PESCOÇO COMO APRESENTAÇÃO INICIAL DE MIELOMA MÚLTIPLO}

${ }^{1}$ Department of Radiology of the Clinics Hospital of the State University of Campinas, Faculty of Medical Sciences, Campinas SP, Brazil; ${ }^{2}$ Department of Pathology of the Clinics Hospital of the State University of Campinas, Faculty of Medical Sciences, Campinas SP, Brazil. 

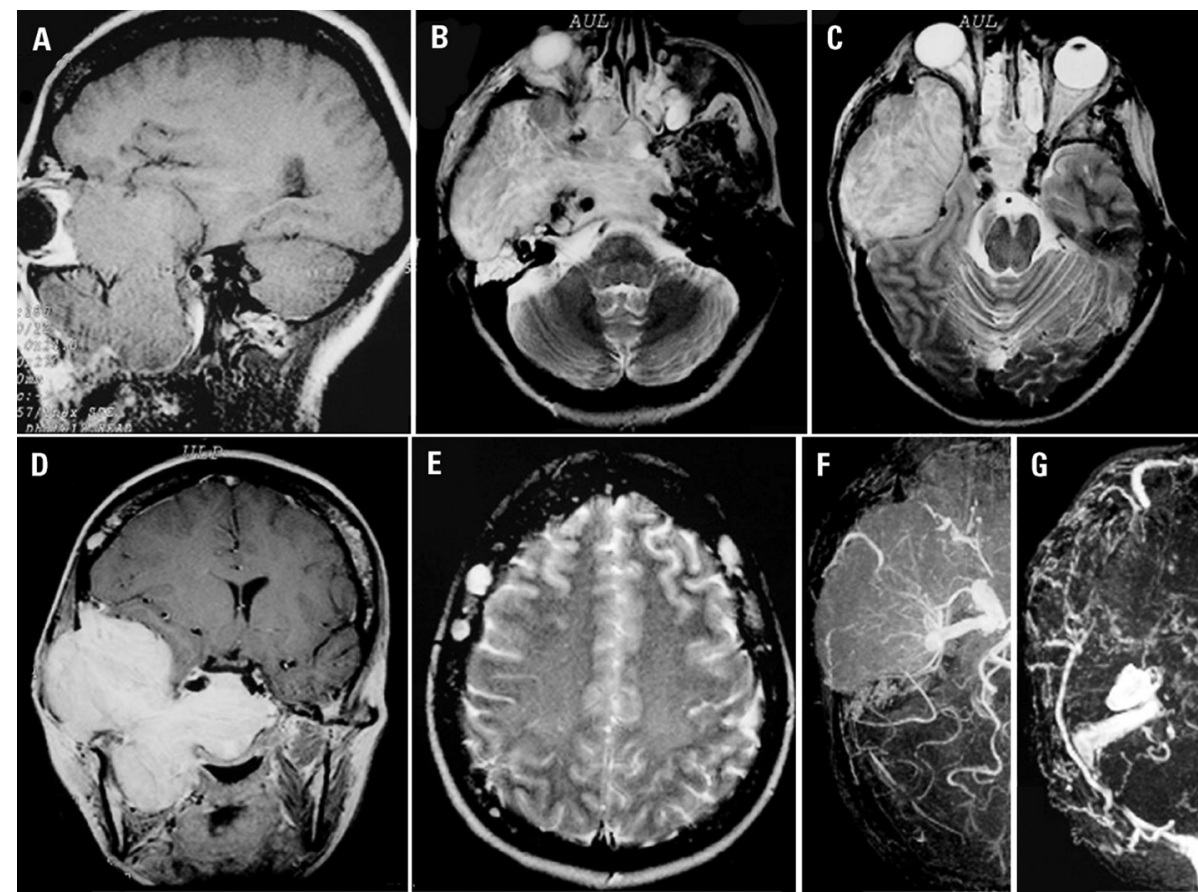

Fig 2. MR: [A] Sagital T1W, [B and C] Axial T2W, [D] Contrast-enchanced Coronal T1W, [E] Axial T2W, [F] Arterial MR Angiography and [G] Venous MR Angiography. Isointensity was noted on T1-weighted images, hyperintensity on T2-weighted and FLAIR images, intralesional flow-voids and strong contrast enhancement. There was bone remodeling and compression of adjacent structures. Lytic bone lesions with no definite margins were seen in the masticator spaces. Other contrast-enhancing nodular intradiploic lesions were also seen. Digital subtraction angiography demonstrated the right external carotid artery as the major nutrient vessel of the mass.
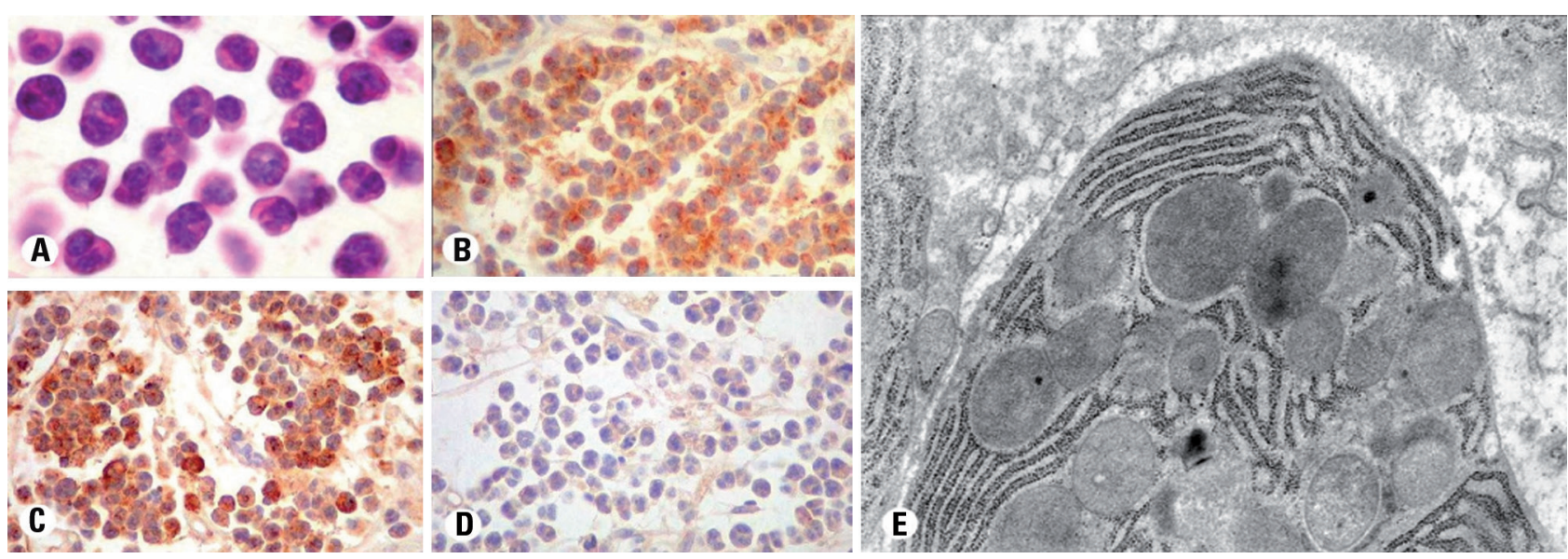

Fig 3. [A] Histological analysis of hematoxilin-eosin stained sections revealed the tumor to be composed of plasma cells with strong basophilic cytoplasm, excentric nuclei and cartwheel chromatin, in a loose capillary-rich stroma. A large proportion of cells contained two to four nuclei. Mitotic figures were very rare and necrosis was not seen. [B, C and D] Immunohistochemistry showed exclusive expression of kappa chains, while lambda chains were thoroughly negative. Many cells were positive for CD138, a reliable marker of plasma cells. [E] On electron microscopy most cells contained abundant cisterns of rough endoplasmatic reticulum, often in parallel arrays along the cell periphery. Macrophages in the process of phagocytosis of apoptotic tumor cells were plentiful.

\section{REFERENCES}

1. Lin C, Luciani A, Belhadj K, et al. Patients with plasma cell disorders examined at whole-body dynamic contrast-enhanced magnetic resonance imaging. Radiology 2009;250:905-915.

2. Cerase A, Tarantino A, Gozzetti A, et al. Intracranial involvement in plas- mocytomas and multiple myeloma: a pictorial essay. Neuroradiology 2008;50:665-674

3. Angtuaco JC, Fassas ABT, Walker R, Sethi R, Barlogie B. Multiple myeloma: clinical review and diagnostic imaging. Radiology 2004:231:11-23.

4. Provenzale JM, Schaefer P, Traweek ST, et al. Craniocerebral plasmacytoma: MR features. AJNR Am J Neuroradiol 1997;18:389-392. 